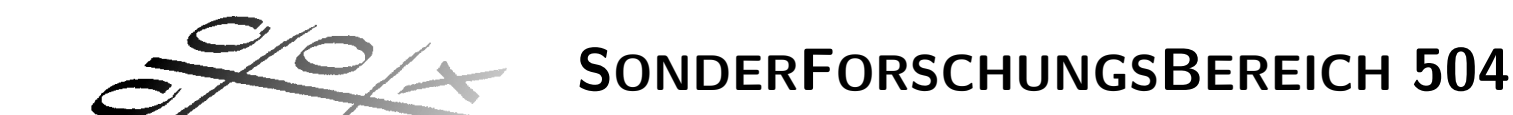

Rationalitätskonzepte,

Entscheidungsverhalten und

ökonomische Modellierung

\begin{tabular}{|} 
No. 08-01 \\
Erwartungsbildung über den Wahlausgang und \\
ihr Einfluss auf die Wahlentscheidung \\
Sascha Huber* \\
and Thomas Gschwend ${ }^{* *}$ \\
and Michael F. Meffert \\
and Franz Urban Pappi****
\end{tabular}

January 2008

Financial support from the Deutsche Forschungsgemeinschaft, SFB 504, at the University of Mannheim, is gratefully acknowledged.

*Sonderforschungsbereich 504, email: shuber@rumms.uni-mannheim.de

**Sonderforschungsbereich 504, email: Thomas.Gschwend@mzes.uni-mannheim.de

*** Sonderforschungsbereich 504, email: meffert@sfb504.uni-mannheim.de

****Sonderforschungsbereich 504, email: fupappi@rumms.uni-mannheim.de

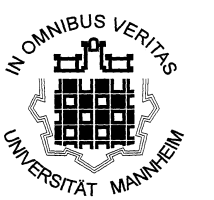

Universität Mannheim

L 13,15

68131 Mannheim 


\title{
Erwartungsbildung über den Wahlausgang und ihr Einfluss auf die Wahlentscheidung
}

\author{
Sascha Huber \\ Mannheimer Zentrum für Europäische \\ Sozialforschung (MZES) \\ Universität Mannheim \\ 68131 Mannheim \\ GERMANY \\ Phone: +49-621-181-2856 \\ Fax: +49-621-181-2845 \\ shuber@rumms.uni-mannheim.de \\ Michael F. Meffert \\ Sonderforschungsbereich 504 \\ Universität Mannheim \\ L 13, 15 \\ 68131 Mannheim \\ GERMANY \\ Phone: +49-621-181-3438 \\ Fax: +49-621-181-3451 \\ meffert@sfb504.uni-mannheim.de
}

\author{
Thomas Gschwend \\ Graduate School of Economic \& \\ Social Sciences (GESS) \\ Universität Mannheim \\ 68131 Mannheim \\ GERMANY \\ Phone: +49-621-181-2087 \\ Fax: +49-621-181-3699 \\ gschwend@uni-mannheim.de \\ Franz Urban Pappi \\ Lehrstuhl für politische Wissenschaft I \\ Universität Mannheim \\ 68159 Mannheim \\ GERMANY \\ Phone: +49-621-181-2810 \\ Fax: +49-621-181-2067 \\ fupappi@rumms.uni-mannheim.de
}

\begin{abstract}
Abstact
Erwartungen über den Wahlausgang haben einen festen Platz sowohl in Rational-Choice-Theorien des Wählerverhaltens als auch in stärker sozialpsychologisch orientierten Ansätzen. Die Bildung von Erwartungen und ihr Einfluss auf die Wahlentscheidung ist dabei jedoch ein noch relativ unerforschtes Gebiet. In diesem Beitrag werden anhand von Wahlstudien für Belgien, Österreich und Deutschland verschiedene Fragen der Erwartungsbildung und ihrer Auswirkungen untersucht. Zunächst wird die Qualität der Gesamterwartungen analysiert und verschiedene Faktoren identifiziert, die einen systematischen Einfluss auf die Erwartungsbildung haben. Im zweiten Schritt wenden wir uns den Einzelerwartungen über verschiedene Parteien und Koalitionen zu und finden eine moderate Verzerrung zugunsten der präferierten Parteien und Koalitionen. Dabei kann gezeigt werden, dass der Effekt des Wunschdenkens mit dem politischen Wissen und dem Bildungsgrad abnimmt. Schließlich werden in einem letzten Schritt zwei unterschiedliche Logiken für die Auswirkungen von Erwartungen getestet, das rationale Kalkül des koalitionsstrategischen Wählens zur Vermeidung der Stimmenvergeudung sowie der sozialpsychologisch begründete BandwagonEffekt. Das Ausmaß an politischem Wissen scheint dabei eine zentrale vermittelnde Variable zwischen den beiden Logiken zu sein.
\end{abstract}




\section{Sascha Huber, Thomas Gschwend, Michael F. Meffert, Franz Urban Pappi}

\section{Erwartungsbildung über den Wahlausgang und ihr Einfluss auf die Wahlentscheidung}

\section{Einleitung}

Wahlumfragen und Projektionen der jüngsten Vergangenheit, sowohl zur vergangenen Bundestagswahl in Deutschland als auch zur vergangenen Nationalratswahl in Österreich waren, gemessen am tatsächlichen Wahlergebnis, nicht besonders gut. Eine mögliche Erklärung in Zeiten abnehmender Parteibindungen und einer zunehmend volatilen Wählerschaft könnte eine stärkere Rolle von Erwartungen bei der Wahlentscheidung sein. Wenn Wähler ihre Wahlentscheidung nicht nur an ihren Präferenzen über die politischen Parteien und Kandidaten festmachen, sondern auch an ihren Erwartungen über den Wahlausgang und diese Erwartungen gerade durch Meinungsumfragen mitbestimmt werden, dann werden Wahlumfragen und Projektionen, die diesen Prozess nicht mit einbeziehen und oft nicht einbeziehen können, ungenauer.

In Theorien zum Wählerverhalten haben Erwartungen über den Wahlausgang seit langem ihren festen Platz. Im Rational-Choice Ansatz wird davon ausgegangen, dass Wähler ihren Nutzen aus der Regierungstätigkeit ziehen. Deshalb spielen bei einer rationalen Wahl nicht nur die Präferenzen der Wähler eine Rolle, sondern immer auch ihre Erwartungen über den Wahlausgang (Downs 1957; Riker und Ordershook 1968). Wähler, die ihren Erwartungsnutzen maximieren, werden dann je nach Wahlsystem und Wahlsituation auch einmal strategisch eine andere als die präferierte Partei wählen (Cox 1997). Erwartungen über den Wahlausgang spielen aber auch in eher sozialpsychologisch orientierten Ansätzen der Wahlforschung eine Rolle. Erwartungen über den Wahlgewinner oder Wahlverlierer werden in diesen Ansätzen als direkte Entscheidungsgrundlage, relativ unabhängig von den Präferenzen, konzipiert. Im Resultat wird es deshalb zu Bandwagon oder Underdog - Effekten kommen, die lediglich auf den Erwartungen über den Wahlausgang basieren (z.B. Simon 1954; Bartels 1985).

Trotz der zentralen Rolle, die Erwartungen in diesen verschiedenen Ansätzen der Wahlforschung spielen, ist die Erwartungsbildung zum Wahlausgang ein noch relativ unerforschtes Gebiet. Eine dominante Stellung in der bisherigen Forschung zur Erwartungsbildung nehmen Befunde zum so genannten "wishful thinking" ein. 
Studien in verschiedenen Ländern konnten zeigen, dass Wähler die Wahlchancen ihrer bevorzugten Partei oder ihres bevorzugten Kandidaten systematisch überschätzen (z.B. Granberg und Brent 1993; Uhlaner und Grofman 1986; Abramson et al 1992; Babad 1997; Schoen 1999). Darüber hinaus herrscht Uneinigkeit wie gut Wähler ihre Erwartungen vor Wahlen an exogene Informationen wie Wahlumfragen anpassen (Lewis-Beck und Skalaban 1989; Gimpel und Harvey 1997; Irwin und van Holsteyn 2002; Blais und Bodet 2006; Faas und Schmitt-Beck 2007). In unserem Beitrag zur Erwartungsbildung in Österreich, Deutschland und Belgien wollen wir versuchen, über das Nebeneinanderstellen von Wunschdenken auf der einen Seite und der Anpassung an Wahlumfragen auf der anderen Seite hinaus zu gehen und mögliche Faktoren aufzeigen, die einen systematischen Einfluss auf die Qualität der Erwartungsbildung ausüben. Die Qualität der Erwartungsbildung wird dabei nicht, wie häufig in bisherigen Studien, nur an den Erwartungen über die Wahlchancen jeweils einer Partei festgemacht, sondern an den Gesamterwartungen für verschiedene Parteien und mögliche Koalitionen. In einem zweiten Schritt sollen dann auch die Einzelerwartungen für die Parteien und ihre mögliche Verzerrung durch Präferenzen untersucht werden. Dabei steht die Frage im Mittelpunkt, ob Wunschdenken durch politisches Wissen und Bildung reduziert wird. Ein dritter Analyseabschnitt wendet sich dann den Erwartungen über einzelne Koalitionen zu. Es wird geprüft, ob auch hier Wunschdenken eine Rolle spielt und Koalitionspräferenzen einen eigenständigen Effekt auf die Koalitionserwartungen ausüben. Im letzten Abschnitt werden die Auswirkungen der Erwartungen auf die Wahlentscheidung anhand von drei Beispielen analysiert. Der Einfluss von Erwartungen wird dabei sowohl für ein rationales Kalkül der Maximierung des Erwartungsnutzens getestet als auch für einen sozialpsychologischen direkten Effekt. Auf Seiten des rationalen Kalküls werden die Strategien der Vermeidung von Stimmenvergeudung und des koalitionsstrategischen Wählens, auf Seiten des sozialpsychologisch direkten Einflusses von Erwartungen der Bandwagon-Effekt geprüft. Dabei wird politisches Wissen als vermittelnde Variable vorgeschlagen um zu erklären, von wem Erwartungen nach einem rationalen Kalkül eingesetzt werden und von wem als direkte Entscheidungshilfe für die Wahlentscheidung.

\section{Daten}

Drei Wahlstudien sind Grundlage unserer verschiedenen Analysen. Für Belgien wurde die Vorwahlbefragung 2003 verwendet, die in unserem Auftrag von ipsos Belgium durchgeführt wurde. Darin wurden 1825 zufällig ausgewählte belgische Wahlberechtigte im Zeitraum vom 2.5. bis 17.5.2003 telefonisch befragt. Für Österreich wurde die Vorwahlbefragung zur Nationalratswahl 2006 verwendet, die 
in unserem Auftrag von Infratest dimap durchgeführt wurde. Darin wurden 1501 zufällig ausgewählte österreichische Wahlberechtigte im Zeitraum vom 18.9. bis 30.9. 2006 telefonisch befragt. Für Deutschland können wir dankenswerter Weise auf die Studie "Kampagnendynamik 2005" von Rüdiger Schmitt-Beck und Thorsten Faas zurückgreifen ${ }^{1}$. Die Vorwahlwelle dieser Studie umfasst 3583 zufällig ausgewählte Wahlberechtigte, die in der Zeit vom 8.8 bis 17.9.2005 befragt wurden. Diese Studie wurde als Rolling Cross-Section konzipiert. Wir verwenden sie bei unseren Analysen jedoch jeweils als Querschnitt.

\section{Wovon hängt die Qualität der Erwartungsbildung über den Wahlausgang ab?}

Die Qualität der Erwartungsbildung kann als möglichst große Übereinstimmung der eigenen Erwartungen mit der bestmöglichen Informationsquelle, repräsentativen Wahlumfragen, konzipiert werden. Im folgenden werden vier Faktoren vorgestellt, die einen systematischen Einfluss auf diese so verstandene Erwartungsqualität ausüben sollten: die rationale Abwägung des Aufwandes zur Erwartungsbildung, der Einfluss von Parteiidentifikation, der soziale Kontext, sowie schließlich individuelle Unterschieden an politischem Wissen, Interesse und Bildung. Im den nächsten Abschnitten wird kurz auf jeden der vier Faktoren und die verwendeten empirischen Indikatoren in den drei untersuchten Ländern eingegangen.

(i) Rationale Abwägung des Aufwandes: Das Bilden von Erwartungen über den Wahlausgang ist aufwändig. Wähler werden wohl meist keine intrinsische Freude daran haben, Wahlumfragen zu konsultieren. Das Sammeln von Informationen sollte daher eher von instrumentellem Nutzen sein. Nach einem rationalen Kalkül sollten Wähler einen umso größeren Aufwand zur Erwartungsbildung betreiben, je relevanter Erwartungen über den Wahlausgang für die eigene Wahlentscheidung sind. Einen indirekten Beleg für eine generelle Abwägung des Aufwandes bei der Informationssuche zu Wahlen liefert Larcinese (2007). Er kann für Großbritannien zeigen, dass die Nachfrage nach Informationen über Wahlen mit der Knappheit der Wahlentscheidung im Wahlkreis steigt.

Als empirischen Indikator für ein rationales Abwägen des Aufwandes zur Erwartungsbildung verwenden wir in unserer Untersuchung zum einen die Distanz zwischen der erstpräferierten und der zweitpräferierten Partei. Je größer der Nutzenunterschied ist zwischen diesen beiden Parteien, desto geringer fällt in einem einfachen Erwartungsnutzenmodell der Wahlentscheidung der

${ }^{1}$ ZA-Studiennummer 4302. 
Erwartungsterm ins Gewicht. Mit einer wachsenden Distanz zwischen Erstpräferenz und Zweitpräferenz sollte ceteris paribus also die Qualität der Erwartungen zurückgehen. Wer denkt, nur durch das Wirken einer bestimmten Partei Nutzen zu erfahren und überhaupt keinen Nutzen durch das Wirken anderer Parteien, braucht keine aufwändige Erwartungsbildung. Wer dagegen verschiedene Parteien ähnlich beurteilt, sollte einen größeren Nutzen in der Erwartungsbildung sehen. Ein weiterer Indikator für eine rationale Abwägung des Aufwandes für die Erwartungsbildung ist die Wahrscheinlichkeit der Wahlbeteiligung. Je geringer die Wahrscheinlichkeit der Wahlbeteiligung ist, desto geringer sollte auch der Aufwand für die Erwartungsbildung sein. Es wird also erwartet, dass die Qualität der Erwartungsbildung mit der Wahrscheinlichkeit der Wahlbeteiligung steigt. Ein dritter hier benutzter Indikator ist die subjektiv wahrgenommene Alternativlosigkeit der eigenen Wahlabsicht. Wer sich nicht vorstellen kann, eine andere als die präferierte Partei zu wählen, der sollte auch keinen Aufwand bei der Erwartungsbildung betreiben. Folglich sollte er auch weniger genaue Erwartungen über den Wahlausgang bilden.

(ii) Parteiidentifikation: Häufig wurde eine Verzerrung der Erwartungen über die Wahlchancen einzelner Parteien nachgewiesen. Dieser Effekt des Wunschdenkens, wonach Wähler die Wahlchancen ihrer bevorzugten Partei oder ihres bevorzugten Kandidaten systematisch überschätzen, legt den Schluss nahe, dass Wähler, die sich grundsätzlich mit einer Partei identifizieren, auch eine generell stärker verzerrte Erwartungsbildung über die Wahlaussichten von allen Parteien und Koalitionen haben. Ist dies der Fall, sollten Befragte mit Parteiidentifikation schlechtere Gesamterwartungen bilden als Befragte ohne Parteiidentifikation. Auf der anderen Seite spricht aber auch einiges für einen umgekehrten Zusammenhang. Es kann argumentiert werden, dass Wähler, die langfristig einer bestimmten Partei zuneigen und somit eine stärkere Bindung an das politische System haben, eine stärkere Motivation haben und sich leichter Erwartungen über den Wahlausgang bilden sollten. Als "member of the polity" (Lewis-Beck and Skalaban 1989: 153) sollten sie eher vertraut sein mit den politischen Kräfteverhältnissen. Ist dies der Fall, sollten Wähler mit Parteiidentifikation bessere Gesamterwartungen bilden als Wähler ohne Parteiidentifikation.

(iii) Sozialer Kontext: Unabhängig von der Konsultation von Wahlumfragen bietet der soziale Kontext Wählern eine heuristische Informationsquelle über die Stimmungslage im Land. Wie in anderen Bereichen der politischen Meinungsbildung wird sich der soziale Kontext von Wählern deshalb auch auf die Erwartungsbildung auswirken (Lazarsfeld et al 1944; Huckfeldt und Sprague 1995). Wenn sich im eigenen Bekanntenkreis viele für die Wahl von Partei A entscheiden, kann man versucht sein, dies als Indikator für die Wahlchancen von Partei A im ganzen Land sehen. Lemert (1986) fand einen großen Einfluss von 
Freunden und Verwandten bei der Erwartungsbildung zu einem Referendum, größer sogar als der Einfluss von Meinungsumfragen. Ähnlich bieten sich Berichte in lokalen Medien über die lokale Stimmungslage an. Daschmann (2000) konnte experimentell zeigen, dass Berichte über die persönlichen Wahlabsichten einzelner Bürger einen größeren Einfluss auf die Erwartungen von Versuchspersonen über den Wahlausgang haben als die numerischen Informationen von Wahlumfragen. Wenn lokale Medien bei ihren Berichten über die Wahlabsichten einzelner Bürger repräsentativ vorgehen, sollten die typischen Wahlabsichten einer Region in den lokalen Medien stärker vertreten sein. Entsprechend würde man folgenden Zusammenhang zwischen regionalem Kontext und der Qualität der Erwartungsbildung erwarten: in einem regionalen Meinungsumfeld, das stark vom durchschnittlichen Meinungsumfeld im Land abweicht, sollte es schwerer sein, Erwartungen über den nationalen Wahlausgang zu bilden. Es wird deshalb erwartet, dass die Qualität der Erwartungen mit der regionalen Diskrepanz vom nationalen Wahlergebnis abnimmt. Als empirischer Indikator soll in Österreich die aufsummierte Abweichung des Landesergebnisses der einzelnen fünf größten Parteien in der Nationalratswahl vom Bundesergebnis dienen ${ }^{2}$. Im Fall von Deutschland ist die Diskrepanz vor allem eine zwischen Ostdeutschland und Westdeutschland. Die Landesergebnisse der Bundestagswahl im Osten - mit einer sehr starken Linken - weichen deutlich stärker vom Bundesergebnis ab als in den westdeutschen Bundesländern. Deshalb wird hier als Indikator eine einfache Dummy-Variable für Ostdeutschland verwendet. Da in Belgien in den unterschiedlichen Landesteilen unterschiedliche Parteien zur Wahl stehen, wird sowohl die Abweichung des Landesergebnis vom Bundesergebnis nach dem österreichischen Muster eingeführt als auch für den wallonischen Landesteil kontrolliert. In allen drei Ländern wird für die Sonderfälle der Städte Berlin, Wien, und Brüssel kontrolliert.

(iv) Politisches Wissen, Interesse und Bildung: Es erscheint sehr plausibel anzunehmen, dass die Qualität der Erwartungsbildung von individuellen Unterschieden der Wähler bezüglich ihres politischen Wissens, ihres Bildungsgrades, und ihres allgemeinen politischen Interesses beeinflusst wird. Wähler mit höherem politischem Wissen ${ }^{3}$ sollten auch über ein größeres Wissen bezüglich der Stimmungslage im Land verfügen. Wählern mit höherer Bildung

2 Ideale Indikatoren für den Einfluss des sozialen Kontexts wären natürlich Netzwerkdaten. Da diese aber nicht vorliegen, erscheinen die hier verwendeten Indikatoren allerdings die, wenn auch beschränkten so doch, bestmöglichen zu sein.

3 Die Variable des politischen Wissens wurde in den drei Ländern folgendermaßen gebildet. In Österreich wurden vier Fragen zum politischen Wissen gestellt: die Höhe der Arbeitslosigkeit, Wissen um Wahlkreiskandidaten, Mehrheit im Bundesrat, und die 4 Prozent - Hürde. Für jede richtige Antwort wurde der Wert eins zugewiesen, für jede falsche und für jede nicht gegebene der Wert null. Die Variable des politischen Wissens wurde dann durch das einfache Aufsummieren dieser Werte gebildet. In Belgien gab es drei Wissensfragen. Abgefragt wurde, welches Amt Isabelle Durant inne hatte und welches Amt Frank Vandenbroucke. Zudem wurde nach der Partei von Filip Dewinter gefragt. In Deutschland wurde lediglich eine Frage zum politischen Wissen gestellt, nämlich die nach der Mehrheit im Bundesrat. 
sollte es unter Umständen leichter fallen, Informationen über die Wahlaussichten einzelner Parteien einzuordnen, und wer sich schließlich besonders für Politik interessiert, sollte auch ein Interesse am wahrscheinlichen Ausgang der nächsten Wahl haben. In der Literatur gibt es allerdings allgemeine Zweifel, ob Wähler mit höherem politischem Wissen, Interesse oder Bildung grundsätzlich zu "besseren" politischen Urteilen kommen (vgl. z.B. Luskin 2002; Tetlock 2005). Auch speziell für die Qualität der Erwartungsbildung gibt es widersprüchliche Befunde, ob Wissen, Interesse oder Bildung einen positiven Einfluss auf die Erwartungen ausüben (Lewis-Beck und Skalaban 1989; Babad 1995; Dolan und Holbrook 2001). Dabei wurde jedoch bisher vor allem für die Erwartungen zu einzelnen Parteien getestet. Hier soll nun geprüft werden wie sich die individuellen Unterschiede auf die die Qualität der Gesamterwartungen auswirken ${ }^{4}$.

In Bezug auf den Einfluss des politischen Interesses soll zudem noch ein weiterer Zusammenhang getestet werden. In der Literatur wurde die Tendenz herausgestellt, dass in den vergangenen Jahren bei der Berichterstattung über Wahlen stärker der sportlicher Wettlaufcharakter hervorgehoben wird und weniger politische Kontroversen (Brettschneider 1996, 2000). Folgt man der Logik, dass dieser so genannte "Horse-Race" Journalismus der Wahlkampfberichterstattung auf eine Nachfrage auf Seiten der Leser stößt, dann sollte es eine Abkopplung zwischen einem allgemeinen politischen Interesse und dem Interesse am Wettlaufcharakter des Wahlkampfes geben. Ein Interesse am Wahlkampf hat danach nicht unbedingt mit originär politischem Interesse zu tun. Daraus lässt sich die testbare Hypothese ableiten, dass das Interesse am Wahlkampf einen größeren Einfluss auf die Qualität der Erwartungsbildung haben sollte als ein allgemeines politisches Interesse. Umgekehrt sollte dann gelten: Wenn es zu keiner signifikanten Abkopplung des allgemeinen politischen Interesses von einem Interesse am Wettlaufcharakter des Wahlkampfes gekommen ist, sollte das Interesse am Wahlkampf keinen größeren Einfluss auf die Erwartungsqualität haben.

Bei der Schätzung unseres Modells zur Erwartungsqualität in Österreich, Deutschland und Belgien wird neben den oben genannten unabhängigen Variablen außerdem für die demographischen Variablen Alter und Geschlecht kontrolliert. Als abhängige Variable wurde aus den verschiedenen Fragen zu den Wahlchancen der Parteien und potentiellen Koalitionen ein Index der Erwartungsqualität gebildet. Dabei wurde jede gestellte Frage in den Datensätzen zu den Wahlchancen der Parteien und den möglichen Mehrheiten danach kodiert, ob die angegebene Antwort den Wahlumfragen entspricht oder nicht. Wer beispielsweise im deutschen Fall erwartet hat, dass die Grünen "bestimmt" oder "wahrscheinlich"

\footnotetext{
${ }^{4}$ Ein möglicher vermittelnder Mechanismus zwischen den individuellen Unterschieden bei Wissen, Bildung und Interesse und der Erwartungsqualität ist die Kenntnisnahme von Wahlumfragen. Für Österreich liegt eine Variable über die Häufigkeit der Kenntnisnahme von Wahlumfragen vor und wird entsprechend in das Modell eingeführt.
} 
genügend Stimmen bekommen werden, um in den Bundestag einzuziehen, erhält für diese Frage den Wert eins. Wer eine andere oder keine Antwort gibt, erhält den Wert null. Der Index der Erwartungsbildung ergibt sich dann aus der Aufsummierung der zugewiesenen Werte für die einzelnen Fragen. In Deutschland sind das vier Fragen (drei Fragen über die Wahlchancen von Parteien und eine über die erwarteten Koalitionen), in Belgien sind es drei Fragen (eine Frage nach der Wahlchance einer Partei, eine nach den Mehrheitsaussichten für die präferierte Koalition und eine nach dem Sitzanteil dieser Koalition). Für Österreich kann auf sechs Fragen zu den Chancen der verschiedenen Parteien und sieben Fragen zu den Chancen der verschiedenen denkbaren Koalitionen zurückgegriffen werden.

Bevor auf die erklärenden Faktoren der Erwartungsbildung in den drei Ländern eingegangen wird, soll zunächst kurz der Blick auf die unterschiedlichen Niveaus der Erwartungsqualität in den drei Ländern gelenkt werden. Vergleicht man die verschiedenen Wahlsysteme, sollten Wähler in Deutschland und Österreich einen direkteren Nutzen darin sehen, über die Wahlchancen der Parteien und Koalitionen informiert zu sein, als Wähler in Belgien. Während in Deutschland und Österreich Wähler recht einfach die erwarteten Stimmanteile proportional in erwartete Sitze übersetzen können, ist die Übersetzung der Stimmanteile in Sitze in Belgien komplizierter. Zusätzlich wird Wählern in Belgien die schwierige Regierungsbildung nach der Wahl bewusst sein, bei der nicht nur die Mehrheitsverhältnisse zwischen den Parteifamilien entscheiden, sondern auch der Konflikt zwischen den zwei Landesteilen. Es ist deshalb zu vermuten, dass Wähler in Belgien einen insgesamt geringeren Aufwand bei der Erwartungsbildung betreiben. Vergleicht man nun die Erwartungsqualität in den drei Ländern, findet sich der erwartete Unterschied. Auf der einen Seite stehen Österreich und Deutschland mit einem recht hohen Mittelwert der Erwartungsqualität. In einem Intervall von null bis eins ist der Mittelwert der Erwartungsqualität in Österreich .70 (Standardabweichung .19), in Deutschland findet sich ein Mittelwert von .66 (Standardabweichung .31). In Belgien ist der Mittelwert mit .30 (Standardabweichung: .24) weniger als halb so groß. Diese Unterschiede entsprechen den Erwartungen bezüglich der Wirkungen des Wahlsystems, können wegen der unterschiedlichen Zusammensetzung der Indizes aber natürlich nur vorsichtig gebraucht werden.

Tabelle 1 enthält die OLS - Modelle der Qualität der Erwartungsbildung in Österreich, Deutschland und Belgien. Alle Variablen in den Modellen wurden auf ein Intervall von null bis eins normiert. Angegeben werden jeweils die unstandardisierten Regressionskoeffizienten. Leere Zellen zeigen an, dass in dem betroffenen Land kein entsprechender Indikator zur Verfügung stand. 
Tabelle 1: Die Qualität der Gesamterwartung in Österreich, Deutschland und Belgien

\begin{tabular}{|c|c|c|c|}
\hline & Österreich & Deutschland & Belgien \\
\hline \multicolumn{4}{|l|}{ Rationale Abwägung des } \\
\hline $\begin{array}{l}\text { Distanz von Erstpräferenz zur } \\
\text { Zweitpräferenz } \\
\text { Wahrscheinlichkeit der } \\
\text { Wahlbeteiligung }\end{array}$ & $\begin{array}{l}-.13 * * * \\
(.02) \\
.08 * * * \\
(.02)\end{array}$ & $\begin{array}{l}-.14 * * * \\
(.03) \\
.12 * * * \\
(.03)\end{array}$ & $\begin{array}{l}-.05 * * \\
(.02)\end{array}$ \\
\hline Wahl alternativlos & & $\begin{array}{l}-.03^{* *} \\
(.01)\end{array}$ & \\
\hline \multicolumn{4}{|l|}{ Parteiidentifikation } \\
\hline $\begin{array}{l}\text { PID: Ja: } 1 \text { Nein: } 0 \\
\text { Für Belgien: } \\
\text { Bevorzugte Partei: Ja: } 1 \text { Nein: } 0\end{array}$ & $\begin{array}{l}.02 * * \\
(.01)\end{array}$ & $\begin{array}{l}.06^{* * * *} \\
(.01)\end{array}$ & $\begin{array}{l}.03^{* *} \\
(.01)\end{array}$ \\
\hline \multicolumn{4}{|l|}{$\begin{array}{l}\text { Politisches Wissen, Bildung, } \\
\text { Interesse }\end{array}$} \\
\hline Politisches Wissen & $\begin{array}{l}.10^{* * * *} \\
(.02)\end{array}$ & $\begin{array}{l}.15^{* * * *} \\
(.01)\end{array}$ & $\begin{array}{l}.12^{* * * *} \\
(.02)\end{array}$ \\
\hline Bildung & $\begin{array}{l}.05^{* * *} \\
(.01)\end{array}$ & $\begin{array}{l}.13^{* * *} \\
(.02)\end{array}$ & $\begin{array}{l}.06^{* *} \\
(.02)\end{array}$ \\
\hline Politisches Interesse & $\begin{array}{l}.05^{* *} \\
(.02)\end{array}$ & $\begin{array}{l}.27 * * * \\
(.02)\end{array}$ & \\
\hline Interesse an Wahlkampf & $\begin{array}{l}.03 \\
(.02)\end{array}$ & $\begin{array}{l}.03 \\
(.02)\end{array}$ & \\
\hline Wahrnehmung von Wahlumfragen & $\begin{array}{l}.05^{* * * *} \\
(.02)\end{array}$ & & \\
\hline $\begin{array}{l}\text { Sozialer Kontext } \\
\text { Abweichung des Landesergebnis } \\
\text { vom Bundesergebnis }\end{array}$ & $\begin{array}{l}-.05^{* *} \\
(.02)\end{array}$ & & $\begin{array}{l}-.01 \\
(.03)\end{array}$ \\
\hline $\begin{array}{l}\text { Region: } \\
\text { Ostdeutschland } \\
\text { bzw. Wallonien }\end{array}$ & & $\begin{array}{l}-.02^{*} \\
(.01)\end{array}$ & $\begin{array}{l}.01 \\
(.01)\end{array}$ \\
\hline $\begin{array}{l}\text { Stadt: } \\
\text { Wien bzw. Berlin bzw. Brüssel }\end{array}$ & $\begin{array}{l}.03^{*} \\
(.01)\end{array}$ & $\begin{array}{l}.04 \\
(.03)\end{array}$ & $\begin{array}{l}-.01 \\
(.02)\end{array}$ \\
\hline \multicolumn{4}{|l|}{ Demographische Merkmale } \\
\hline Geschlecht: männlich & $\begin{array}{l}.02 * * \\
(.01)\end{array}$ & $\begin{array}{l}.12^{* * *} \\
(.01)\end{array}$ & $\begin{array}{l}.01 \\
(.01)\end{array}$ \\
\hline Alter & $\begin{array}{l}-.09 * * * \\
(.02)\end{array}$ & $\begin{array}{l}.03 \\
(.02)\end{array}$ & $\begin{array}{l}-.06 * * \\
(.02)\end{array}$ \\
\hline $\mathrm{R}^{2}$ & .15 & .31 & .05 \\
\hline $\mathrm{N}$ & 1301 & 3300 & 1769 \\
\hline
\end{tabular}

Zellen enthalten die unstandardisierten Regressionskoeffizienten. Alle Variablen wurden auf ein Intervall von null bis eins normiert. * $\mathrm{p}<.10 * * \mathrm{p}<.05 * * * \mathrm{p}<.01$ 
Der erste untersuchte Faktor, die rationale Abwägung des Aufwandes zur Erwartungsbildung, bestätigt sich in den drei Modellen sehr gut. Die Indikatoren gehen durchweg in die erwartete Richtung und sind signifikant. Je größer der Abstand zwischen Erstpräferenz und Zweitpräferenz, desto geringer ist die Erwartungsqualität und je sicherer sich Wähler sind, zur Wahl zu gehen, desto besser wird die Erwartungsqualität. Zudem zeigen Wähler, die ihre Wahl als alternativlos sehen, eine geringere Erwartungsqualität. Wähler scheinen also tatsächlich den Aufwand, den sie bei ihrer Erwartungsbildung betreiben, abzuwägen. Wähler, für die die Erwartungen in ihrem Entscheidungskalkül eine geringere Rolle spielen sollten, zeigen wie erwartet eine geringere Erwartungsqualität. Die Befunde sind dabei, soweit sie zu testen waren, robust über alle drei Länder.

Ein verzerrender Effekt des zweiten Faktors, der Parteiidentifikation, ist nicht festzustellen. Ganz im Gegenteil haben Wähler mit Parteiidentifikation signifikant bessere Gesamterwartungen über den Wahlausgang als Wähler ohne Parteiidentifikation. Dieser Befund ist insofern erstaunlich, als er in Kontrast zu einer Vielzahl von Studien steht, die suggerieren, dass der einzige systematische Effekt bei der Erwartungsbildung der verzerrende Effekt des Wunschdenkens ist. Untersucht man aber nicht nur die Erwartungen für eine einzelne Partei, sondern wie hier die Qualität der Erwartungen über verschieden Parteien und Koalitionen, ergibt sich der umgekehrte Effekt. Parteianhänger haben bessere Gesamterwartungen, in allen drei untersuchten Ländern. Der Effekt des Wunschdenkens scheint also nicht so sehr auf die Gesamterwartungen durchzuschlagen. Vielmehr ist davon auszugehen, dass Wähler mit einer Parteiidentifikation besser an das politische System angebunden sind, eine größere politische Motivation besitzen und als "member of the polity" geringere Kosten haben, die politische Stimmungslage einzuschätzen.

Die Effekte des sozialen Kontexts gehen in allen drei Ländern in die erwartete Richtung, in Österreich und Deutschland sind sie signifikant. Je stärker das Landesergebnis vom Bundesergebnis in Österreich abweicht, desto geringer wird die Erwartungsqualität. Entsprechend ergibt sich in Deutschland für die neuen Bundesländer mit ihren stark abweichenden politischen Kräfteverhältnissen ein negativer Effekt. In Belgien sind die Effekte des regionalen Kontextes dagegen gering. Sowohl die Effekte für die Abweichung vom Landesergebnis als auch die Kontrolle für Wallonien erreichen keine Signifikanz. Bedenkt man die Tatsache, dass in Belgien in unterschiedlichen Landesteilen unterschiedliche Parteien zur Wahl stehen und Wähler deshalb besser daran gewöhnt sein sollten, dass ihr sozialer Kontext nicht unbedingt repräsentativ für das Land ist, erstaunt dieses Ergebnis kaum. Um einen Hinweis auf die Größe des Effekts in den anderen beiden Ländern zu bekommen, bietet sich der Vergleich mit dem Einfluss der Kenntnisnahme von Wahlumfragen in Österreich an. Die Koeffizienten für den Einfluss des Konsums von Wahlumfragen und für den Einfluss des regionalen 
Kontexts sind in Österreich ähnlich groß. Obwohl die Indikatoren für den sozialen Kontexts nicht optimal sind, zeigt sich also ein nicht geringer Effekt.

Die insgesamt stärksten Effekte in den Modellen ergeben sich für die Indikatoren des politischen Wissens, der Bildung und des politischen Interesses. Je größer das politische Wissen, je höher der Bildungsgrad und je stärker das allgemeine politische Interesse ist, desto besser sind auch die Erwartungen über den Wahlausgang. Dabei zeigt sich für das politische Wissen in allen drei Ländern ein starker positiver Einfluss. Wer politisch sehr informiert ist, wird auch eher über die Wahlchancen der verschiedenen Parteien und Koalitionen informiert sein. Auch die Befunde zum Bildungsgrad sind eindeutig für alle drei Länder. ${ }^{5}$ Interessant ist auch der Vergleich der Effekte von allgemeinem politischen Interesse und spezifischem Interesse am Wahlkampf. Sollte der so genannte "Horse-Race" Journalismus dazu geführt haben, dass sich Wähler im Wahlkampf vor allem für den Stand beim Wettlauf-Spektakel des Wahlkampfes interessieren und weniger für die allgemeine Politik, sollten Wähler, die sich besonders für den Wahlkampf interessieren, aber auch bessere Erwartungen über den Wahlausgang haben. Die Daten bestätigen das aber nicht. Allgemeines politisches Interesse hat einen deutlichen signifikanten Einfluss auf die Qualität der Erwartungsbildung in den untersuchten Ländern, ein besonderes Interesse am Wahlkampf hatte dagegen keinen signifikanten Einfluss.

Erstaunlicherweise finden sich auch für die demographischen Variablen des Alters und des Geschlechts systematische und signifikante Effekte. Das Alter hat in Österreich und Belgien einen negativen Einfluss auf die Erwartungsbildung, das männliche Geschlecht in Deutschland und Österreich einen positiven Einfluss. Als Grund für den negativen Effekt des Alters kann vielleicht das Ausbilden einer Wahlroutine mit dem Alter angeführt werden. Wer sein Leben lang Partei A gewählt hat und das auch für den Rest seines Lebens vorhat, der braucht sich auch wenig um die Wahlchancen der Parteien zu kümmern. Den Einfluss des Geschlechts können wir uns dagegen nicht erklären.

Insgesamt ergeben sich für alle untersuchten vier Faktoren über alle drei Länder hinweg sehr stabile Zusammenhänge mit der Erwartungsqualität. Lediglich in Belgien konnten keine Effekte für einen der Faktoren gefunden werden. Diese stabilen Zusammenhänge in den drei untersuchten Ländern sprechen dafür, dass die Faktoren einen systematischen Einfluss auf die Qualität der Erwartungsbildung in Mehrparteiensystemen ausüben. Insbesondere mit der rationalen Abwägung des Aufwandes zur Erwartungsbildung konnte ein bisher völlig unbeachteter Faktor identifiziert werden. Der positive Einfluss, den unsere Analyse für die Parteiidentifikation zeigt, steht in einem gewissen Kontrast zu früheren Studien, die vor allem auf die verzerrenden Effekte des Wunschdenkens durch Parteiidentifikation hingewiesen haben, widerspricht diesen aber nicht unbedingt.

\footnotetext{
${ }^{5}$ Die Effekte der Bildung, des politischen Interesses und des politischen Wissens scheinen dabei nicht nur auf einer stärkeren Kenntnisnahme von Wahlumfragen zu basieren, wie das Modell für Österreich zeigt.
} 
Während die Identifikation mit einer Partei die Gesamterwartungen über den Wahlausgang verbessert, kann sie trotzdem zu mehr oder weniger großen Verzerrungen bei der Einschätzung der Wahlchancen der eigenen Partei führen. Diesen potentiellen Verzerrungen widmet sich der nächste Abschnitt.

\section{Erwartungen für einzelne Parteien: Reduziert sich Wunschdenken mit dem politischen Wissen und dem Bildungsgrad?}

Bei den Erwartungen über einzelne Parteien lässt sich häufig ein Wunschdenken finden. Danach bewerten Wähler die Wahlchancen ihrer bevorzugten Parteien oder Kandidaten systematisch höher als dies vom Durchschnitt der Wählerschaft getan wird. Dabei geht es nicht wie oben darum, ob die Erwartungen gemäß der Umfragen "korrekt" oder "falsch" sind, sondern darum, dass Anhänger von Parteien die Wahlchancen ihrer Partei grundsätzlich höher einschätzen. Dieser Effekt des Wunschdenkens ist vielfach und für unterschiedliche Länder nachgewiesen worden (vgl. z.B. Lazarsfeld et al. 1944: 106; Granberg und Brent 1983; Granberg und Holmberg 1988; Uhlaner und Grofman 1986; Babad und Yacobos 1993; Mutz 1998; Schoen 1999; Blais und Turgeon 2004)6. Unklar scheint dagegen, ob der Effekt für alle Wähler stabil ist oder ob vermittelnde Variablen eine Rolle spielen. Einerseits finden sich in einer Reihe von Studien sehr robuste Effekte des Wunschdenkens. Danach verkleinert sich der Effekt nicht signifikant, wenn besondere Anreize zur Genauigkeit gegeben werden oder wenn Befragte dazu angehalten werden "objektiv" zu sein (Babad und Katz 1991; Babad 1997). Genauso hatte hohes politisches Wissen in einer Studie zu Wahlen in Israel keinen signifikant reduzierenden Effekt auf das Wunschdenken (Babad 1995). Auf der anderen Seite fanden jedoch Lewis-Beck und Skalaban (1989) einen positiven Effekt von Bildung auf die Genauigkeit von Erwartungen über die Wahlchancen einzelner Parteien und Dolan und Holbrook (2001) konnten für Wahlen in den USA eine signifikante Reduzierung des Wunschdenkens durch politisches Wissen zeigen. Neben dem empirischen Interesse, wie verbreitet Wunschdenken tatsächlich ist, ist die Frage einer möglichen Reduzierung des Wunschdenkens auch für eine theoretische Fundierung des Effekts relevant. Wenn sich keine Reduktion finden lässt, kann dies als Hinweis auf rein motivationale Prozesse (vgl. Kunda 1990) des Wunschdenkens interpretiert werden. Wenn sich dagegen eine Reduktion findet, kann dies als Hinweis auf zusätzliche kognitive Effekte verstanden werden.

\footnotetext{
${ }^{6}$ Interessanterweise scheint der Effekt des Wunschdenkens bei der Erwartungsbildung im politischen Bereich im Vergleich zu anderen Bereichen besonders stark ausgeprägt zu sein (Price 2000)
} 
Da der Effekt des Wunschdenkens für alle politischen Parteien und ihre Anhänger gleich sein sollte, wurde für jedes Land ein Modell für alle Parteien, zu denen Daten über die Erwartungen vorliegen, geschätzt. Als abhängige Variable dienen die Erwartungen über die Wahlchancen der jeweils präferierten Parteien, wobei ein höherer Wert höher erwartete Wahlchancen angibt. Für Österreich liegen die Erwartungen für alle fünf größeren Parteien vor, für Deutschland nur die Erwartungen über die Wahlchancen von FDP, Grüne und Linkspartei und für Belgien nur die Erwartungen über das Abschneiden der VLD. Daraus ergibt sich für Österreich ein fünffach und für Deutschland ein dreifach "gestapelter" Datensatz. Da jeder Befragte in Deutschland und Österreich also mehrere (drei bzw. fünf) Bewertungen zu dem Datensatz beiträgt, sind die Bewertungen nicht unabhängig. Folglich werden alle Antworten eines Befragten als "Cluster" behandelt und robuste Standardfehler, die für diese Cluster korrigieren, geschätzt ${ }^{7}$.

Erste unabhängige Variable ist die Erstpräferenz für die fragliche Partei, die aus dem Vergleich der Skalometerwerte der Parteien gebildet wurde. Zweite unabhängige Variable ist der Skalometerwert der fraglichen Parteien: damit lässt sich testen ob Wähler auch unabhängig von ihrer Erstpräferenz verzerrte Erwartungen bilden, je nachdem ob Ihnen eine Partei mehr oder weniger zusagt. Um zu testen ob sich Wunschdenken mit dem Bildungsgrad und dem politischen Wissen verkleinert, werden Interaktionsterme dieser Variablen mit dem Skalometerwert der Partei eingeführt. Da für Belgien lediglich die Erwartungen über die VLD abgefragt wurden, diese Partei aber nur im niederländisch sprechenden Teil antritt, wurde für den Landesteil kontrolliert.

Tabelle 2 zeigt einen deutlichen signifikanten Effekt für die Erstpräferenz. Die Wahlchancen für die bevorzugte Partei werden systematisch höher eingeschätzt. Es zeigt sich aber auch ein zusätzlicher Effekt für den Skalometerwert der Parteien: je mehr Wähler, unabhängig von ihrer Erstpräferenz, von einer Partei halten, desto besser schätzen sie auch deren Wahlchancen ein. Umgekehrt lässt sich der Zusammenhang natürlich auch so formulieren: je weniger von einer Partei gehalten wird, desto eher werden die Wahlchancen im Vergleich zum Durchschnitt unterschätzt. Die Haupteffekte für Bildung und Wissen sind in Deutschland und Österreich signifikant positiv. Das ist insofern erwartungsgemäß, da die Fragen in beiden Fällen so formuliert waren, dass nach den Wahlumfragen ein höherer Wert auf der Skala auch wahrscheinlicher war. So wurde in Deutschland zum Beispiel danach gefragt, wie sicher der Einzug der drei kleinen Parteien eingeschätzt wird. Nach den Wahlumfragen war der Einzug der drei kleinen Parteien recht sicher, deshalb ist auch ein positiver Haupteffekt für die Variablen Bildung und Wissen zu erwarten. In Belgien dagegen war die wahrscheinliche Kategorie bei der Frage

\footnotetext{
${ }^{7}$ Wegen fehlender Angaben tragen nicht alle Befragten exakt gleich viele Bewertungen bei.
} 
nach den Wahlchancen der VLD eine Mittelkategorie. Deshalb war auch kein Effekt für Bildung und Wissen zu erwarten ${ }^{8}$.

Tabelle 2: Wunschdenken und Erwartungen über die Wahlchancen einzelner Parteien

\begin{tabular}{|c|c|c|c|}
\hline & $\begin{array}{c}\text { Österreich } \\
\text { (Erwartungen über } \\
\text { die Wahlchancen von } \\
\text { ÖVP, SPÖ, Grüne, } \\
\text { FPÖ, BZÖ) }\end{array}$ & $\begin{array}{c}\text { Deutschland } \\
\text { (Erwartungen über } \\
\text { die Wahlchancen von } \\
\text { FDP, Grüne, Linke) }\end{array}$ & $\begin{array}{c}\text { Belgien } \\
\text { (Erwartungen über } \\
\text { die Wahlchancen der } \\
\text { VLD) }\end{array}$ \\
\hline Erstpräferenz für die Partei & $\begin{array}{l}.17^{* *} \\
(.07)\end{array}$ & $\begin{array}{l}.49 * * * \\
(.08)\end{array}$ & $\begin{array}{l}.38^{* *} \\
(.15)\end{array}$ \\
\hline Skalometerwert der Partei & $\begin{array}{l}.16^{* * *} \\
(.02)\end{array}$ & $\begin{array}{l}.22 * * * \\
(.02)\end{array}$ & $\begin{array}{l}.16^{* *} \\
(.06)\end{array}$ \\
\hline Politisches Wissen & $\begin{array}{l}.20 * * * \\
(.04)\end{array}$ & $\begin{array}{l}.99 * * * \\
(.05)\end{array}$ & $\begin{array}{l}-.02 \\
(.06)\end{array}$ \\
\hline Bildung & $\begin{array}{l}.11^{* * *} \\
(.02)\end{array}$ & $\begin{array}{l}.18 * * * \\
(.02)\end{array}$ & $\begin{array}{l}.04 \\
(.03)\end{array}$ \\
\hline $\begin{array}{l}\text { Politisches Wissen X } \\
\text { Skalometerwert der Partei } \\
\text { Bildung X Skalometerwert } \\
\text { der Partei } \\
\text { Wallonien und franz. } \\
\text { Brüssel } \\
\end{array}$ & $\begin{array}{l}-.025 * * * \\
(.006) \\
-.015 * * * \\
(.003)\end{array}$ & $\begin{array}{l}-.052 * * * \\
(.015) \\
-.014 * * \\
(.005)\end{array}$ & $\begin{array}{l}.00 \\
(.02) \\
-.01 \\
(.01) \\
.48^{* * *} \\
(.13) \\
\end{array}$ \\
\hline N (Bewertungen) & 7162 & 10085 & 1283 \\
\hline $\mathrm{N}$ (Befragte) & 1472 & 3473 & 1283 \\
\hline
\end{tabular}

Theoretisch interessanter sind die Interaktionseffekte des Skalometerwerts der Parteien mit Bildung und Wissen. Für Belgien ergibt sich kein Effekt. Für Deutschland und Österreich zeigt sich dagegen ein signifikanter negativer Effekt: Das heißt, je mehr politisches Wissen Wähler haben und je gebildeter sie sind, desto geringer wird das Wunschdenken. Wunschdenken scheint in Deutschland und Österreich also besonders bei Wählern mit geringerer Bildung und geringerem politischen Wissen verbreitet. Dieser Befund spricht gegen eine rein motivationale Begründung von Wunschdenken, denn dann sollte das Ausmaß des Wunschdenkens nicht mit dem Wissen und der Bildung variieren. Ein möglicher kognitiver Zusatz zum Wunschdenken könnte sein, dass Wähler, die weniger "objektive" Informationen über den Wahlausgang haben, und sich unsicher sind, auf ihre eigene Präferenz oder die Präferenzen ihrer sozialen Umfelds zurückgreifen, um von diesen Präferenzen auf die Wahlchancen zu schließen.

${ }^{8}$ Unerwartet ist der starke Effekt der anderen Kontrollvariable in Belgien. Wähler in Wallonien und französischsprachige Wähler in Brüssel schätzen die Wahlchancen der VLD deutlich besser ein als die Wähler im flämischen Landesteil. 


\section{Wunschdenken und Koalitionserwartungen}

Der Effekt des Wunschdenkens wurde bisher immer nur für die Erwartungen von einzelnen Parteien überprüft. Erwartungen über Koalitionen wurden dagegen nicht behandelt. Dabei spricht einiges dafür, dass Wähler nicht nur Präferenzen über Parteien haben, sondern auch Koalitionen durchaus eigenständig bewerten (vgl. Pappi und Gschwend 2005; Gschwend 2007, Meffert und Gschwend 2007). Entsprechend ist zu erwarten, dass sich der Effekt des Wunschdenkens auch bei der Erwartungsbildung über mögliche Koalitionen finden lassen sollte. Wer eine bestimmte mögliche Koalition einer anderen vorzieht, sollte die Chancen dieser Koalition, eine Mehrheit zu erreichen, überschätzen. Hier soll getestet werden, ob dies der Fall ist oder ob Koalitionsbewertungen so stark hinter Parteipräferenzen nachgelagert sind, dass sich der Effekt des Wunschdenkens nicht findet. Weiterhin soll getestet werden, inwieweit auch hier Wunschdenken mit dem Bildungsgrad und dem politischen Wissen abnimmt.

Als abhängige Variable dienen im österreichischen Fall die Erwartungen über die fünf möglichen Koalitionen ÖVP/SPÖ, ÖVP/FPÖ, ÖVP/BZÖ, ÖVP/Grüne und SPÖ/Grüne, in Deutschland die Erwartungen über die Koalitionen Union/FDP, Union/SPD und SPD/Grüne. Für Belgien liegen nur Daten über die Mehrheitserwartungen der bevorzugten Koalition vor, weshalb kein Test von Wunschdenken möglich ist. Da der Effekt des Wunschdenkens für alle Koalitionen der gleiche sein sollte, wird wie oben ein Gesamtmodell für alle Koalitionen geschätzt. Daraus ergibt sich für Österreich wiederum ein fünffach und für Deutschland ein dreifach "gestapelter" Datensatz. Die verschiedenen Bewertungen eines Befragten werden wiederum als Cluster behandelt und es werden robuste Standardfehler, die für diese Cluster korrigieren, werden geschätzt.

Tabelle 3 zeigt einen starken signifikanten Effekt für Wunschdenken bei der Erwartungsbildung zu Koalitionen: Wähler mit der Präferenz für eine bestimmte Koalition schätzen die Chancen dieser Koalition höher ein als Wähler mit einer anderen oder keiner Präferenz. Der Effekt tritt dabei unter Kontrolle der Parteipräferenzen für die beiden jeweils beteiligten Koalitionspartner auf. Das heißt, Wunschdenken bei den Koalitionserwartungen ist nicht durch vorgelagerte Parteipräferenzen der beteiligten Koalitionspartner bedingt, sondern ein eigenständiger Effekt. Wie im Fall von Parteipräferenzen lässt sich ein Interaktionseffekt der Koalitionspräferenz mit der Bildung bzw. mit dem politischen Wissen beobachten ${ }^{9}$. In Deutschland sind beide Interaktionseffekte

${ }^{9}$ Die starken Haupteffekte von Bildung und Wissen in Deutschland lassen sich damit erklären, dass in dem deutschen Datensatz nicht für jede mögliche Koalition erhoben wurde, in welchem Ausmaß sie erwartet wird, sondern mit einer multiple-response Frage lediglich erhoben wurden, welche Koalition erwartet wurde. Das heißt, wer überhaupt eine der drei plausiblen Koalitionen angab, hatte im Durchschnitt eine höheres Wissen und Bildungsgrad als solche Befragte, die angaben eine andere Koalition, etwa eine Alleinregierung der Union, zu erwarten. In Österreich erklärt sich 
signifikant, für Österreich nur der Effekt der Bildung. Es scheint also, wie bei den Parteierwartungen, einen systematischen negativen Zusammenhang zwischen dem Ausmaß an Wunschdenken und dem Bildungsgrad bzw. dem politischen Wissen von Bürgern zu geben.

Tabelle 3: Wunschdenken und Erwartungen für einzelne Koalitionen

\begin{tabular}{|c|c|c|}
\hline & \begin{tabular}{l}
\multicolumn{1}{c}{ Österreich } \\
Erwartungen für Koalitionen \\
ÖVP/SPÖ, ÖVP/FPÖ, \\
ÖVP/BZÖ, ÖVP/Grüne, \\
SPÖ/Grüne)
\end{tabular} & \begin{tabular}{l}
\multicolumn{1}{c}{ Deutschland } \\
Erwartungen für Koalitionen \\
Union/FDP, Union/SPD, \\
SPD/Grüne
\end{tabular} \\
\hline Koalitionspräferenz & $\begin{array}{l}1.79 * * * \\
(.14)\end{array}$ & $\begin{array}{l}1.85^{* * * *} \\
(.20)\end{array}$ \\
\hline Parteipräferenz 1. Koalitionspartner & $\begin{array}{l}.15^{* * *} \\
(.05)\end{array}$ & $\begin{array}{l}.07 \\
(.05)\end{array}$ \\
\hline Parteipräferenz 2. Koalitionspartner & $\begin{array}{l}.86 * * * \\
(.07)\end{array}$ & $\begin{array}{l}-.17^{*} \\
(.08)\end{array}$ \\
\hline Politisches Wissen & $\begin{array}{l}.07 * * * \\
(.02)\end{array}$ & $\begin{array}{l}.75^{* * * *} \\
(.07)\end{array}$ \\
\hline Bildung & $\begin{array}{l}.09 * * * \\
(.01)\end{array}$ & $\begin{array}{l}.12^{* * *} \\
(.02)\end{array}$ \\
\hline $\begin{array}{l}\text { Politisches Wissen X } \\
\text { Koalitionspräferenz }\end{array}$ & $\begin{array}{l}.04 \\
(.05)\end{array}$ & $\begin{array}{l}-.49 * * * \\
(.15)\end{array}$ \\
\hline Bildung X Koalitionspräferenz & $\begin{array}{l}-.16^{* * *} \\
(.03)\end{array}$ & $\begin{array}{l}-.14^{* * *} \\
(.05)\end{array}$ \\
\hline N (Bewertungen) & 7136 & 10590 \\
\hline N (Befragte) & 1453 & 3530 \\
\hline
\end{tabular}

\section{Erwartungen und ihr Einfluss auf die Wahlentscheidung}

Welche Rolle spielen die Erwartungen, so sie denn gebildet sind, nun bei der Wahlentscheidung? Sowohl in Theorien rationalen Wählens als auch in stärker sozialpsychologisch orientierten Ansätzen der Wahlforschung werden Erwartungen als ein Faktor von Wahlentscheidung gesehen. In den folgenden zwei Abschnitten sollen drei Beispiele den Einfluss der Erwartungen auf die Wahlentscheidung sowohl nach einem rationalen Mechanismus als auch nach einem sozialpsychologisch-heuristischen Mechanismus illustrieren. Als mögliche Fälle

der, wenngleich geringere, Haupteffekt dadurch dass alle abgefragten Koalitionen, bis auf die Koalition von OVP und BZÖ, aufgrund der Umfragen eine gewisse Plausibilität hatten. 
eines rationalen Einbeziehens der Erwartungen wird die Wahl der Linkspartei in Deutschland und die Wahl des BZÖ in Österreich untersucht. Als Fall eines möglichen sozialpsychologisch-heuristischen Mechanismus - des BandwagonEffekts - wird die Wahl der ÖVP in Österreich analysiert. Dabei wird auch untersucht, ob bestimmte Wählergruppen eher als andere der einen oder anderen Logik folgen. Falls es Effekte für die verschiedenen Mechanismen gibt, wird erwartet, dass Wähler mit größerem politischen Wissen eher einer rationalen Logik folgen und Wähler mit geringerem politischem Wissen eher der heuristischen. Wähler mit größerem politischen Wissen sollten eher gewillt und in der Lage sein, aus ihren Präferenzen und Erwartungen einen Erwartungsnutzen zu kalkulieren, Wähler mit geringerem politischen Wissen sollten dagegen auf einfachere, heuristische Art ihre Erwartungen in die Wahlentscheidung einbeziehen ${ }^{10}$.

Bei der Analyse der Wirkung von Erwartungen auf die Wahlentscheidung ist dabei jeweils das oben gezeigte Wunschdenken bei der Erwartungsbildung zu beachten. Dieses Wunschdenken erschwert den Test, ob Erwartungen tatsächlich einen Einfluss auf die Wahlentscheidung haben. Möglich ist auch, dass der vermeintliche Einfluss der Erwartungen lediglich die Präferenzen bzw. deren Intensität widerspiegelt ${ }^{11}$. In den folgenden Analysen wird deshalb versucht, möglichst genau für die Präferenzen und deren Intensität zu kontrollieren.

\subsection{Rationale Mechanismen des Einflusses von Erwartungen auf die Wahlentscheidung: Vermeidung von Stimmenvergeudung und koalitionsstrategisches Kalkül}

Um die potentielle rationale Einbeziehung von Erwartungen auf die Wahlentscheidung zu testen, bietet sich die Analyse der Wahl der Linkspartei in Deutschland und die Wahl des BZÖ in Österreich an. Für die Wahl der Linkspartei lassen sich zwei mögliche rationale Logiken finden, nach denen die Erwartungen eine Rolle spielen sollten. Erstens sollten mögliche Wähler der Linkspartei bedenken, ob sie ihre Stimme vergeuden, wenn sie sie der Linkspartei geben, diese

${ }^{10}$ Es ist nicht grundsätzlich davon auszugehen, dass weniger kenntnisreiche Wähler generell stärker auf Heuristiken zurückgreifen. Manche Heuristiken für die Wahlentscheidung werden vor allem von Wählern mit hohem politischem Wissen genutzt (vgl. Lau und Redlawsk 2006). Das gilt vor allem für Heuristiken, für deren Nutzung selbst ein gewisses politisches Wissen notwendig ist. Für den Bandwagon-Effekt, so er denn eine Heuristik darstellt, erscheint es allerdings plausibel anzunehmen, dass er eine Rückfalloption für Wähler mit geringem politischem Wissen darstellt.

11 Noch schwieriger wird der Test, wenn die Daten keine Messung der Präferenzen sondern nur die Wahlabsicht enthalten. Unklar bleibt dann, ob die Wahlabsicht durch die Erwartungen beeinflusst wurde oder ob die Erwartungen durch die Wahlabsicht beeinflusst wurden. Ein Vorteil der hier verwendeten Daten ist, dass über die Skalometerwerte ein Präferenzmaß vorliegt. 
dann aber womöglich nicht ins Parlament einzieht (vgl. z.B. Cox 1997). Um ihre Stimme nicht zu verschwenden, sollten Wähler die Linke umso eher wählen, je sicherer sie sich sind, dass die Linke den Einzug ins Parlament auch schaffen kann. Trifft dieses wasted-vote Argument zu, sollte sich nach Kontrolle der Präferenzen und deren Intensität ein Effekt der Erwartungen auf die Wahlentscheidung zugunsten der Linken finden lassen.

Die zweite mögliche Logik bezieht sich auf koalitionsstrategisches Wählen und ist weniger intuitiv. Für koalitionsstrategisches Wählen sind drei Aspekte entscheidend: die Präferenzen über die Koalitionen, Erwartungen über den Wahlausgang und von den Parteien ausgesendete Koalitionssignale für die Regierungsbildung. Linhart (2007) zeigt für bestimmte Konstellationen von Koalitionssignalen für die Bundestagswahl 2005 eine koalitionsstrategische Logik zur Wahl der Linkspartei auf. Wenn Wähler eine große Koalition bevorzugen, die Möglichkeit einer Linkskoalition aus SPD, Grünen und Linke aufgrund der Koalitionssignale ausschließen, und die Möglichkeit der Bildung einer großen Koalition aber sehen, dann kann für sie die Wahl der Linken die rationale Wahl sein: eine möglichst starke Linkspartei verhindert dann alle möglichen anderen Zweier-Koalitionen bis auf die große Koalition. Nach diesen Überlegungen lassen sich drei Hypothesen für die Wirkung von Koalitionspräferenzen und Koalitionserwartungen für die Wahl der Linkspartei ableiten: Die Wahl der Linken sollte wahrscheinlicher werden, erstens wenn Befragte eine große Koalition präferieren, zweitens wenn sie nicht an die Bildung einer Linkskoalition aus SPD, Grünen und Linke glauben und drittens wenn sie die Bildung einer großen Koalition für möglich halten ${ }^{12}$.

Wie Tabelle 4 zeigt, findet sich klare Evidenz für das Vermeiden einer Stimmenvergeudung. Unter Kontrolle der Erstpräferenz für die Linkspartei, den Skalometerwerten für die Linkspartei und für Oskar Lafontaine, der Stärke der Parteiidentifikation und von Ostdeutschland, hat die Erwartung über den Einzug einen signifikanten Effekt auf die Wahl der Partei ${ }^{13}$. Wer also die Chancen der Linkspartei, in den Bundestag einzuziehen, höher einschätzt, der wird sie eher wählen. Gilt dieses Ergebnis nur für die politisch Kenntnisreichen? Um Hinweise auf diese Frage zu bekommen, wurden gesonderte Modelle für Wähler mit größerem politischen Wissen und Wähler mit geringerem politischem Wissen geschätzt $^{14}$. In beiden Modellen ergeben sich für die Strategie der Vermeidung

12 Der rationale Wähler bei Linhart (2007) akzeptiert die Koalitionssignale direkt. Manipuliert werden in seinem Modell lediglich die Koalitionssignale. Hier werden diese Manipulationen in die variablen Koalitionserwartungen der Wählerschaft übersetzt. Die Daten zu den Koalitionserwartungen im deutschen Datensatz sind insofern ideal, da die Erwartungen über die Bildung tatsächlicher Koalitionen nach der Wahl abgefragt werden und nicht nur die Mehrheitserwartungen für verschiedene Parteikonstellationen.

${ }^{13}$ Aus der Analyse werden die Befragten ausgeschlossen, die keine Angabe zu ihrer Wahlabsicht machen oder angeben, bestimmt nicht zur Wahl zu gehen.

${ }^{14}$ Im deutschen Datensatz findet sich leider nur eine Frage zum politischen Wissen, nämlich die nach der Mehrheit im Bundesrat, weshalb keine gleichteiligere Einteilung der 
einer Stimmenvergeudung recht ähnliche Effekte. Es gibt also keine Hinweise darauf, dass Wähler mit geringem politischem Wissen seltener eine Strategie der Vermeidung von Stimmenvergeudung anwenden als Wähler mit hohem politischem Wissen.

Tabelle 4: Erwartungen und die Wahl der Linkspartei bei der Bundestagswahl 2005

\begin{tabular}{llcc}
\hline & $\begin{array}{c}\text { Wahl der Linkspartei } \\
\text { (gesamt) }\end{array}$ & $\begin{array}{c}\text { Wahl der Linkspartei } \\
\text { (hohes politisches } \\
\text { Wissen) }\end{array}$ & $\begin{array}{c}\text { Wahl der Linkspartei } \\
\text { (niedriges politisches } \\
\text { Wissen) }\end{array}$ \\
\hline Erwartung Einzug der & $.38^{* * *}$ & $.34^{* *}$ & $.39^{*}$ \\
Linkspartei & $(.12)$ & $(.15)$ & $(.22)$ \\
Koalitionserwartung: & $.71^{* * *}$ & $.95^{* * *}$ & .33 \\
Union und SPD & $(.27)$ & $(.33)$ & $(.71)$ \\
Koalitionserwartung: SPD, & -.72 & -1.53 & -.01 \\
Grüne und Linke & $(.97)$ & $(1.56)$ & $(1.34)$ \\
Koalitionspräferenz: Union & $.87^{* * *}$ & $.67^{* *}$ & .39 \\
und SPD & $(.29)$ & $(.30)$ & $(.82)$ \\
Erstpräferenz: Linkspartei & $1.70^{* * *}$ & $1.99 * * *$ & $\left(.18^{* *}\right.$ \\
Skalometer Linkspartei & $(.26)$ & $(.30)$ & $.44^{* * *}$ \\
Stärke der & $.53^{* * *}$ & $.59^{* * *}$ & $(.12)$ \\
Parteiidentifikation mit der & $(.07)$ & $(.08)$ & $.65^{* * *}$ \\
Linkspartei & $.50^{* * *}$ & $.42^{* * *}$ & $(.15)$ \\
Skalometer: Lafontaine & $(.09)$ & $(.11)$ & .08 \\
Ostdeutschland & $.11^{* *}$ & $.11^{*}$ & $(.09)$ \\
\hline Pseudo-R & $(.05)$ & $(.06)$ & .06 \\
N & .14 & .26 & $(.44)$ \\
\hline Z & $(.24)$ & $(.29)$ & .57 \\
\hline
\end{tabular}

Zellen enthalten die unstandardisierten Koeffizienten der logistischen Regression.

Standardfehler in Klammern. ${ }^{*} \mathrm{p}<.10 * * \mathrm{p}<.05 * * * \mathrm{p}<.01$

Neben dem Effekt für die wasted-vote Logik finden sich auch für die koalitionsstrategische Logik deutliche Effekte: sowohl die Koalitionspräferenz für eine große Koalition als auch die Erwartung für das Bilden einer großen Koalition erhöht im Gesamtmodell die Wahrscheinlichkeit einer Wahl der Linkspartei signifikant. Der dritte erwartete Zusammenhang im Sinne der Koalitionsstrategie, der Einfluss der Erwartungen über das Bilden einer Linkskoalition aus SPD, Grünen und Linke geht in die erwartete negative Richtung, erreicht aber keine Signifikanz. Dieses klare Ergebnis für ein koalitionsstrategisches Kalkül ist

Gruppen möglich war: 2469 Befragte wussten die Antwort, 1087 gaben die falsche Antwort oder keine Antwort. 
insofern erstaunlich, als es doch einen recht elaborierten Umgang der Wähler mit ihren Präferenzen und ihren Erwartungen über Wahlausgang und Regierungsbildung erfordert. Wie die einzeln geschätzten Modelle für Befragte mit hohem und niedrigem politischen Wissen zeigen, sind es auch tatsächlich die politische Kenntnisreicheren, die dieses koalitionsstrategische Kalkül anzuwenden scheinen. Während die Effekte im Modell für politisch kenntnisreiche Befragte erhalten bleiben, gehen sie im Modell für die politisch weniger Kenntnisreichen verloren. Im Gegensatz zur intuitiveren wasted-vote Logik scheint das kompliziertere koalitionsstrategische Kalkül tatsächlich nur von Wählern mit höherem politischem Wissen angewendet zu werden.

Tabelle 5: Erwartungen und die Wahl des BZÖ bei der Nationalratswahl 2006

\begin{tabular}{|c|c|}
\hline & Wahl des BZÖ \\
\hline $\begin{array}{l}\text { Erwartungen über Einzug des } \\
\text { BZÖ ins Parlament }\end{array}$ & $\begin{array}{l}1.37 * * * \\
(.45)\end{array}$ \\
\hline Erstpräferenz: BZÖ & $\begin{array}{l}2.21 * * \\
(.87)\end{array}$ \\
\hline Skalometer BZÖ & $\begin{array}{l}.58 * * * \\
(.18)\end{array}$ \\
\hline $\begin{array}{l}\text { Stärke der Parteiidentifikation mit } \\
\text { BZÖ }\end{array}$ & $\begin{array}{l}1.17^{* * *} \\
(.43)\end{array}$ \\
\hline Skalometer: Haider & $\begin{array}{l}.06 \\
(.11)\end{array}$ \\
\hline $\begin{array}{l}\text { Koalitionspräferenz: ÖVP und } \\
\text { BZÖ }\end{array}$ & $\begin{array}{l}1.98 * * \\
(.85)\end{array}$ \\
\hline $\begin{array}{l}\text { Koalitionspräferenz: ÖVP, FPÖ } \\
\text { und BZÖ }\end{array}$ & $\begin{array}{l}1.65 \\
(1.01)\end{array}$ \\
\hline Kärnten & $\begin{array}{l}1.24 \\
(1.11)\end{array}$ \\
\hline Pseudo-R ${ }^{2}$ & .73 \\
\hline $\mathrm{N}$ & 1200 \\
\hline
\end{tabular}

Ein zweites Beispiel, die Wahl des BZÖ, soll die Logik der Vermeidung einer Stimmvergeudung auch für Österreich testen. Nach den Wahlumfragen zur Nationalratswahl war unklar, ob das BZÖ mehr als vier Prozent der Stimmen erhalten wird und somit den Einzug in den Nationalrat erreicht. Entsprechend sollte sich für das BZÖ ein Effekt der Erwartungen auf die Wahl einstellen: Um ihre Stimme nicht zu verschwenden, sollten potentielle Wähler das BZÖ um so eher wählen, je eher sie einen Einzug des BZÖ in das Parlament erwarten. Um einen möglichen Einfluss der Erwartungen zu testen, wird im logit-Modell für die Wahl des $\mathrm{BZÖ}^{15}$ wiederum für die Erstpräferenz, den Skalometerwert der Partei und von

15 Aus der Analyse werden die Befragten ausgeschlossen, die keine Angabe zu ihrer Wahlabsicht machen oder angeben, bestimmt nicht zur Wahl zu gehen. 
Jörg Haider, für die Stärke der Parteiidentifikation mit dem BZÖ, die Koalitionspräferenzen und das Bundesland Kärnten kontrolliert. Tabelle 5 zeigt die Ergebnisse. Danach haben die Erwartungen über den Einzug des BZÖ ins Parlament einen signifikanten Effekt auf die Wahlentscheidung zugunsten des BZÖ. Wer erwartet, dass das BZÖ nicht ins Parlament einziehen kann, wird dem BZÖ mit einer geringeren Wahrscheinlichkeit die Stimme geben ${ }^{16}$.

\subsection{Sozialpsychologisch - heuristische Mechanismen des Einflusses von Erwartungen auf die Wahlentscheidung: Der Bandwagon - Effekt}

In der sozialpsychologisch orientierten Literatur wird der Einfluss von Erwartungen über den Wahlausgang auf die Wahlentscheidung vor allem unter dem Blickwinkel eines direkten Effekts analysiert. Anders als bei der rationalen Kalkulation eines Erwartungsnutzens und dem indirekten Effekt den Erwartungen dabei spielen, wird vermutet, dass Wähler ihre Erwartungen direkt als Entscheidungsgrundlage einsetzen. Der prominenteste direkte Einfluss von Erwartungen auf die Wahlentscheidung ist dabei der Bandwagon-Effekt, wonach Wähler eher einen Kandidaten oder eine Partei wählen, von der sie denken, dass er oder sie gewinnt (z.B. Simon 1954: 246). Als mögliche Fundierung dieses Effekts kommen zwei verschiedene Mechanismen in Frage. Der erste basiert auf dem möglichen Wunsch von Wählern, einer wahrgenommenen Mehrheitsnorm zu entsprechen bzw. auf Seiten des Gewinners zu stehen (vgl. z.B. Asch 1951). Der zweite Mechanismus sieht die Erwartungen über den Gewinner einer Wahl als mögliche heuristische Informationsquelle. Wähler, die ansonsten über geringe Informationen verfügen, nutzen ihre Wahrnehmung der Mehrheitsmeinung um diese zu imitieren: Was die Mehrheit richtig findet, kann so falsch nicht sein (vgl. z.B. Axsom et al. 1987, Mutz 1998, Schmitt-Beck 1996). Welcher Mechanismus zutrifft, lässt sich mit Umfragedaten nur schwer analysieren, allerdings lassen sich aus den beiden Mechanismen Hypothesen über die Rolle von politischem Wissen ableiten. Trifft der erste Mechanismus eines Wählens nach Mehrheitsnorm zu, sollte das Ausmaß an politischem Wissen keine Rolle spielen. Trifft der zweite Mechanismus einer Entscheidungsheuristik zu, sollte der Effekt vor allem bei politisch weniger kenntnisreichen Wählern zu finden sein.

\footnotetext{
${ }^{16}$ Auf bivariater Ebene findet sich keine Evidenz für Unterschiede bei der Anwendung der wasted-vote Logik zwischen Gruppen mit unterschiedlichem Wissensniveau. Separate multivariate Tests für Kenntnisreiche und weniger Kenntnisreiche, wie für die Linkspartei, waren aufgrund fehlender Varianz nicht möglich: Da relativ wenige Befragte in der Umfrage als Wahlabsicht das BZÖ angaben, variierten verschiedene Variablen nicht mehr, wenn nach dem Wissensniveau aufgegliedert wird.
} 
Die empirische Evidenz für einen Bandwagon-Effekt kommt vor allem aus den angelsächsischen Ländern. Er konnte besonders für amerikanische Vorwahlen (z.B. Bartels 1985), aber auch für die Präsidentschaftswahlen in den USA (z.B. Granberg und Holmberg 1988, Skalaban 1988, Gimpel und Harvey 1997) für Wahlen in Großbritannien (McAllister und Studlar 1991, Nadeau, et al. 1994) und in Kanada (Lanoue und Bowler 1998) gezeigt werden. Auf Wahlen in Mehrparteiensystemen ist die Logik des Bandwagon-Effekts dagegen nicht ohne weiteres zu übertragen. Es ist zunächst zu bestimmen, was genau "gewinnen" in einem Mehrparteiensystem bedeutet. Können auch kleine Parteien eine Wahl "gewinnen", etwa weil sie besonders gut abschneiden und z.B. ihren Stimmanteil von 5 auf 10 Prozent verdoppeln? Oder ist die eigentliche "Wahlgewinnerin" immer eine der großen Parteien, die dann auch den Regierungschef stellt? Wird als Gewinner einer der Spitzenkandidaten gesehen? Oder "gewinnen" am Ende Parteikonstellationen, die sich schon vor der Wahl in klarer Form gegenüber standen? Gewinnt also die derzeitige Koalition gegen die Opposition und umgekehrt? Schmitt-Beck (1996) hat sich bei seiner Analyse der Bundestagswahl 1990 für die letztgenannte Spezifikation entschieden und kann einen BandwagonEffekt zugunsten der damals amtierenden Koalition zeigen. Für die damalige Wahl mit zwei klaren Blöcken scheint diese Spezifikation theoretisch nicht unplausibel. Für andere Wahlen mit weniger klar sich gegenüber stehenden Blöcken erscheint das jedoch weniger plausibel. Die Frage, welche "Einheiten" Wähler in Mehrparteiensystemen als mögliche Gewinner einer Wahl sehen und ihre Wahl entsprechend ausrichten - einzelne Parteien, Spitzenkandidaten oder Koalitionen ist also zunächst immer eine empirische.

Unser Datensatz zur Nationalratswahl in Österreich bietet den Vorteil, diese Frage auch empirisch beantworten zu können. In einer offenen Frage wurden die Befragten gebeten anzugeben, wen sie als Gewinner der Wahl erwarten. Von den 1333 Befragten, die darauf eine Antwort gaben, nannten 76 Prozent eine der beiden großen Parteien ÖVP oder SPÖ, 15 Prozent einen der beiden Kanzlerkandidaten, nur knapp sechs Prozent eine Koalition und knapp drei Prozent eine der kleinen Parteien. Interessant ist auch, dass von den sechs Prozent, die überhaupt eine Koalition nannten, mehr als zwei Drittel die große Koalition als Gewinner angaben. Für diese Wahl lässt sich die Frage, wen die Wähler als potentiellen "Gewinner" assoziieren, also eindeutig beantworten. Es ist mit sehr großer Mehrheit eine der großen Parteien. Fügt man auch die Nennung der Spitzenkandidaten zu den Parteiwerten hinzu, sind es 91 Prozent der Wähler, die die Frage des Gewinnes und Verlierens zwischen den großen Parteien und ihren Spitzenkandidaten entschieden sieht. Ein möglicher Bandwagon-Effekt sollte sich deshalb auch bei der Wahlabsicht zwischen diesen beiden Parteien widerspiegeln. Wer denkt die ÖVP gewinnt die Wahl, sollte eher die ÖVP wählen und wer denkt die SPÖ gewinnt die Wahl, sollte eher die SPÖ wählen. Die Verteilung der Erwartungen über den Wahlgewinner bestimmt dann, welche Partei einen Nutzen aus diesem Effekt zieht. Im hier analysierten Fall ist auch das eindeutig: 76 Prozent 
erwarteten die ÖVP oder deren Spitzenkandidaten als Gewinner, nur 17 Prozent die SPÖ oder deren Spitzenkandidaten. Hier wird sich deshalb auf einen möglichen Bandwagon-Effekt zugunsten der ÖVP konzentriert.

Für unsere Analyse des Bandwagon-Effekts in Österreich wird als abhängige Variable also die Dummy - Variable Wahl der ÖVP gewählt ${ }^{17}$ und möglichst genau für die Präferenzen der Wähler kontrolliert. Dazu werden Erstpräferenz, Skalometerwert und Stärke der Parteiidentifikation mit der ÖVP verwendet sowie die Koalitionspräferenz für eine ÖVP-geführte Koalition, die Kanzlerpräferenz und die generelle Zufriedenheit mit der Regierung. Als unabhängige Variable wird die offene Frage nach dem Wahlgewinner genutzt. Mit dem Wert eins werden alle Nennungen der ÖVP und von Wolfgang Schüssel codiert, mit dem Wert null die restlichen Nennungen. Um einen Hinweis zu bekommen, ob es Unterschiede beim Bandwagon-Effekt zwischen verschiedenen Gruppen gibt, wird neben dem Gesamtmodell für alle Wähler wiederum eines für Wähler mit hohem politischem Wissen und eines mit geringem politischen Wissen geschätzt ${ }^{18}$.

Tabelle 6: Bandwagon Effekt für die Wahl der ÖVP bei den Nationalratswahlen 2006

\begin{tabular}{llcc}
\hline & $\begin{array}{c}\text { Wahl der ÖVP } \\
\text { (gesamt) }\end{array}$ & $\begin{array}{c}\text { Wahl der ÖVP } \\
\text { (hohes politisches } \\
\text { Wissen) }\end{array}$ & $\begin{array}{c}\text { Wahl der ÖVP } \\
\text { (niedriges politisches } \\
\text { Wissen) }\end{array}$ \\
\hline Erwarteter Wahlgewinner & $.68^{* *}$ & .97 & $.76^{* *}$ \\
ÖVP:1 Andere/keine:0 & $(.31)$ & $(.60)$ & $(.37)$ \\
Erstpräferenz ÖVP & $1.86^{* * *}$ & $2.16^{* * *}$ & $1.81^{* * *}$ \\
Skalometer ÖVP & $(.26)$ & $(.42)$ & $(.36)$ \\
Stärke der & $.19^{* * *}$ & $.38^{* * *}$ & .08 \\
Parteiidentifikation : ÖVP & $(.07)$ & $(.12)$ & $(.09)$ \\
Koalitionspräferenz: ÖVP- & $.49^{* * *}$ & $.46^{* * *}$ & $.53^{* * *}$ \\
geführt & $(.06)$ & $(.09)$ & $(.08)$ \\
Kanzlerpräferenz & .34 & $.83^{* *}$ & -.08 \\
Schüssel: 1 , Gusenbauer: -1 & $(.24)$ & $(.37)$ & $1.35)$ \\
Andere/keine: 0 & $1.60^{* * *}$ & $1.60^{* *}$ & $(.45)$ \\
Zufriedenheit mit Regierung & $(.37)$ & $(.80)$ & -.09 \\
\hline Pseudo-R & $-.32^{2 *}$ & $-.76^{* *}$ & $(.26)$ \\
$\mathrm{N}$ & $(.19)$ & $(.31)$ & .61 \\
\hline Zellen enthalten die Koeffizienten der logistischen Regression. Standardfehler in Klammern. \\
$*$ p<.1 ** p<.05 ***p<.01
\end{tabular}

17 Aus der Analyse werden die Befragten ausgeschlossen, die keine Angabe zu ihrer Wahlabsicht machen oder angeben, bestimmt nicht zur Wahl zu gehen.

18 Die Einteilung der beiden Gruppen orientierte sich am Median des Indizes für politisches Wissen. Befragte mit einem Wert von zwei und mehr wurden der Gruppe mit hohem Wissen zugeteilt. 
Im Gesamtmodell von Tabelle 6 zeigt sich ein deutlicher Bandwagon-Effekt zugunsten der ÖVP. Unter Kontrolle der relevanten Präferenzen und der Zufriedenheit mit der Regierung, erhöht sich die Wahrscheinlichkeit einer Wahl der ÖVP, wenn Wähler sie oder ihren Spitzenkandidaten als Wahlgewinner erwarten. Ein Vergleich der separat geschätzten Modelle für Wähler mit hohem und mit niedrigem Wissen zeigt, dass lediglich im Modell der Wähler mit niedrigem politischem Wissen der signifikante Effekt der Erwartungen erhalten bleibt.Im Gegensatz zu dem oben analysierten koalitionsstrategischen Kalkül bei der Wahl der Linkspartei in Deutschland, scheint der Bandwagon-Effekt also vor allem auf die Wähler mit geringerem Wissen zurückzuführen zu sein. Damit gibt die Analyse auch einen Hinweis auf die Plausibilität einer theoretischen Fundierung des Bandwagon-Effekts. Wenn Wähler deshalb für den erwarteten Wahlgewinner stimmen, weil sie einer sozialen Mehrheitsnorm entsprechen wollen oder weil sie auf der Seite der Gewinner stehen wollen, sollte das politische Wissen keinen Unterschied machen. Da das Wissen jedoch einen Unterschied macht, erscheint der zweite oben ausgeführte Mechanismus plausibler: Wähler, die über wenig politische Informationen verfügen, nutzen ihre Erwartung über den Wahlgewinner als günstige Informationsquelle für ihre Wahlentscheidung.

\section{Fazit}

In unserer Analyse haben wir zunächst die Qualität der Gesamterwartungen untersucht und vier Gruppen von Faktoren identifiziert, die einen systematischen Einfluss auf die Erwartungsbildung haben: erstens scheinen Wähler den Aufwand bei der Erwartungsbildung auf eine rationale Art und Weise abzuwägen. Wähler haben umso bessere Erwartungen, je relevanter Erwartungen für ihre Maximierung des Erwartungsnutzens bei Wahlen sind. Zweitens zeigt sich ein positiver Effekt für Wähler mit einer Parteiidentifikation, der möglicherweise auf stärkere Bindungen an das politische System zurückgeführt werden kann. Drittens scheint der soziale Kontext eine Rolle zu spielen: je weniger das regionale Umfeld vom nationalen Durchschnitt abweicht, desto besser sind die Erwartungen. Viertens zeigen sich auch deutliche Auswirkungen individueller Unterschiede bezüglich des Ausmaßes an politischem Wissen, des politischen Interesses und des Bildungsgrades. Die gefundenen Zusammenhänge waren dabei zum großen Teil sehr robust über die drei untersuchten Länder hinweg.

In einem zweiten Schritt haben wir uns den Einzelerwartungen über verschiedene Parteien und Koalitionen zugewandt und eine moderate Verzerrung sowohl zugunsten der präferierten Parteien als auch zugunsten der bevorzugten Koalitionen gefunden. Dabei konnte gezeigt werden, dass der Effekt des Wunschdenkens mit dem politischen Wissen und dem Bildungsgrad abnimmt. 
Schließlich wurden in einem letzten Schritt zwei unterschiedliche Logiken für die Auswirkungen von Erwartungen getestet. Auswirkungen von Erwartungen auf die Wahlentscheidung wurden einerseits für ein rationales Kalkül des koalitionsstrategischen Wählens und der Vermeidung von Stimmenvergeudung gezeigt. Andererseits wurde mit dem Bandwagon auch ein sozialpsychologisch direkter Effekt nachgewiesen. Das Ausmaß an politischem Wissen scheint dabei eine zentrale vermittelnde Variable zwischen den beiden Logiken zu sein. Kenntnisreichere Wähler handeln eher nach der ersten Logik und weniger nach der zweiten, weniger kenntnisreiche Wähler handeln eher nach der zweiten und weniger nach der ersten. Erwartungen über den Wahlausgang bilden sich, wie hier gezeigt wurde, also nicht nur auf systematische Art und Weise heraus, auch die Auswirkungen der Erwartungen auf die Wahlentscheidung scheinen auf systematische Art und Weise zu variieren. Das sind viel versprechende Aussichten für weitere Forschung.

\section{Literatur}

Abramson, Paul R.., John H. Aldrich, Phil Paolino, und David W. Rohde, 1992: 'Sophisticated' Voting in the 1988 Presidential Primaries, American Political Science Review 86: 55-69.

Asch, Solomon E., 1951: Effects of Group Pressure upon the Modification and Distortion of Judgements, S. 177-190 in: Harold S. Guetzkow (Hg.): Groups, Leadership, and Men: Research in Human Relations. Pittsburg: Carnegie Press.

Axsom, Danny, Suzanna Yates, und Shelley Chaiken, 1987: Audience response as a heuristic cue in persuasion, Journal of Personality and Social Psychology 53: 30-40.

Babad, Elisha, 1995: Can Accurate Knowledge Reduce Wishful Thinking in Voters' Predictions of Election Outcomes?, Journal of Psychology 129 : 285-300.

Babad, Elisha, 1997: Wishful thinking among voters: motivational and cognitive influences, International Journal of Public Opinion Research 9: 105-126.

Babad, Elisha, und Yosi Katz, 1991: Wishful thinking - against all odds, Journal of Applied Social Psychology 21: 1821-38.

Babad, Elisha, und Eitan Yacobos, 1993: Wish and reality in voters' predictions of election outcomes, Political Psychology 14: 37-54.

Bartels, Larry M., 1985: Expectations and Preferences in Presidential Nominating Campaigns, American Political Science Review 79: 804-815.

Blais, André, und Mathieu Turgeon, 2004: How Good Are Voters at Sorting Out the Weakest Candidate in their Constituency?, Electoral Studies 23: 455-461.

Blais, André, und Marc-Andre Bodet, 2006: Forming expectations about election chances, Social Science Quarterly 87: 477-493

Brettschneider, Frank, 1996: Wahlumfragen und Medien - Eine empirische Untersuchung der Presseberichterstattung über Meinungsumfragen vor den Bundestagswahlen 1980 bis 1994, Politische Vierteljahresschrift 37: 475-493. 
Brettschneider, Frank, 2000: Demoskopie im Wahlkampf - Leitstern oder Irrlicht? S. 477505 in: Markus Klein, Wolfgang Jagodzinski, Ekkehard Mochmann und Dieter Ohr (Hg.): 50 Jahre Empirische Wahlforschung in Deutschland. Entwicklung, Befunde, Perspektiven, Daten. Wiesbaden: Westdeutscher Verlag.

Cox, Gary W., 1997: Making Votes Count. Strategic Coordination in the World's Electoral Systems. Cambridge: Cambridge University Press

Daschmann, Gregor, 2000: Vox Pop \& Polls: The Impact of Poll Results and Voter Statements in the Media on the Perception of a Climate of Opinion, International Journal of Public Opinion Research 12: 160-181.

Dolan, Kathleen A., und Thomas M. Holbrook, 2001: Knowing Versus Caring: The Role of Affect and Cognition in Political Perceptions, Political Psychology 22: 27-44.

Downs, Anthony, 1957: An Economic Theory of Democracy. New York: Harper Collins.

Faas, Thorsten und Rüdiger Schmitt-Beck, 2007: Wahrnehmung und Wirkungen politischer Meinungsumfragen. Eine Exploration zur Bundestagswahl 2005, S.233-267 in: Frank Brettschneider, Oskar Niedermayer, und Bernhard Weßels (Hg.): Die Bundestagswahl 2005. Analysen des Wahlkampfes und der Wahlergebnisse. Wiesbaden: VS.

Gimpel, James G., und Diane H. Harvey, 1997: Forecasts and Preferences in the 1992 General Election, Political Behavior 19: 157-175.

Granberg, Donald, und Edward Brent. 1983: When Prophecy Bends: The PreferenceExpectation Link in U.S. Presidential Elections, 1952-1980, Journal of Personality and Social Psychology 45: 477-491.

Granberg, Donald, und Sören Holmberg, 1988: The Political System Matters: Social Psychology and Voting Behavior in Sweden and the United States. Cambridge: Cambridge University Press.

Gschwend, Thomas, 2007: Ticket-Splitting and Strategic Voting under Mixed Electoral Rules: Evidence from Germany, European Journal of Political Research 46: 1-23.

Huckfeldt, Robert, und John Sprague, 1995: Citizens, Politics and Social Communication. Cambridge: Cambridge University Press

Irwin, Galen A., und Joop J. Van Holsteyn, 2002: According to the Polls: The Influence of Opinion Polls on Expectations, Public Opinion Quarterly 66: 92-104.

Kunda, Ziva, 1990: The Case for Motivated Reasoning, Psychological Bulletin 108: 480498.

Larcinese, Valentino, 2007: The Instrumental Voter goes to the Newsagent. Demand for Information, Marginality and the Media, Journal of Theoretical Politics 19: 248-276.

Lanoue, David J., und Shaun Bowler, 1998: Picking the Winners: Perceptions of Party Viability and Their Impact on Voting Behavior, Social Science Quarterly 79: 361-377.

Lau, Richard R., und David P. Redlawsk, 2006: How Voters Decide. Information Processing during Election Campaigns. Cambridge: Cambridge University Press.

Lazarsfeld, Paul F., Bernard Berelson, und Hazel Gaudet, 1944: The People's Choice. How the Voter Makes Up His Mind in a Presidential Campaign. New York: Columbia University Press.

Lemert, James B., 1986: Picking the Winners: Politician vs. Voter Predictions of Two Controversial Ballot Measures, Public Opinion Quarterly 50: 208-21.

Lewis-Beck, Michael S., und Andrew Skalaban, 1989: Citizen Forecasting: Can Voters See into the Future?, British Journal of Political Science 19: 146-153.

Linhart, Eric, 2007: Rationales Wählen als Reaktion auf Koalitionssignale am Beispiel der Bundestagswahl 2005, Politische Vierteljahresschrift 48: 461-484.

Luskin, Robert C., 2002: From Denial to Extenuation (and Finally Beyond): Political Sophistication and Citizen Performance, S. 187-216 in: James H. Kuklinski (Hg.): Thinking about Political Psychology. Cambridge: Cambridge University Press. 
McAllister, Ian, und Donley T. Studlar, 1991: Bandwagon, Underdog, or Projection? Opinion Polls and Electoral Choice in Britain, 1979-1987, Journal of Politics 53: 720 41.

Meffert, Michael F., und Thomas Gschwend, 2007: Strategic Voting under Proportional Representation and Coalition Governments: A Simulation and Laboratory Experiment, SFB 504 Discussion Paper No. 07-55, Mannheim: Universität Mannheim.

Mutz, Diana C., 1998: Impersonal Influence: How Perceptions of Mass Collectives Affect Political Attitudes. Cambridge: Cambridge University Press.

Nadeau, Richard, Richard G. Niemi, und Timothy Amato, 1994: Expectations and Preferences in British General Elections, American Political Science Review 88: 37183.

Pappi, Franz Urban, und Thomas Gschwend, 2005: Partei- und Koalitionspräferenzen der Wähler bei den Bundestagswahlen 1998 und 2002, S. 284-305 in: Jürgen W. Falter, Oscar W. Gabriel und Bernhard Wessels (Hg.): Wahlen und Wähler. Wiesbaden: VS.

Price, Paul C., 2000: Wishful thinking in the prediction of competitive outcomes, Thinking and Reasoning 6: 161-172.

Riker, William H., und Peter C. Ordeshook, 1968: A Theory of the Calculus of Voting, American Political Science Review 62: 25-32.

Schmitt-Beck, Rüdiger, 1996: Mass Media, the Electorate, and the Bandwagon. A Study of Communication Effects on Vote Choice in Germany, International Journal of Public Opinion Research 8: 266-291.

Schoen, Harald, 1999: Mehr oder weniger als fünf Prozent - ist das wirklich die Frage?, Kölner Zeitschrift für Soziologie und Sozialpsychologie 51: 565-582.

Simon, Herbert A., 1954: Bandwagon and Underdog Effects and the Possibility of Election Predictions, Public Opinion Quarterly 18: 245-253.

Skalaban, Andrew, 1988: Do Polls Affect Elections? Some 1980 Evidence, Political Behavior 10: 136-150.

Tetlock, Philip E., 2005: Expert Political Judgment: How Good Is It? How Can We Know? Princeton: Princeton University Press.

Uhlaner, Carole J., und Bernard Grofman, 1986: The race may be close but my horse is going to win: Wish fulfillment in the 1980 Presidential Election, Political Behavior 8: 101-128. 


\begin{tabular}{lll}
\hline \hline Nr. & Author & Title
\end{tabular}

08-01 Sascha Huber Thomas Gschwend Michael F. Meffert Franz Urban Pappi

07-76 Michael Bremert Dennis Voeller Nicole Zein

07-75 Jannis Bischof Jens Wüstemann

07-74 Markus Glaser Philipp Schäfers Martin Weber

07-73 Jannis Bischof Michael Ebert

07-72 Susanne Abele Garold Stasser

07-71 Julian Rode Marc Le Menestrel

07-70 Markus Glaser Martin Weber

07-69 Jose Apesteguia Steffen Huck Jörg Oechssler Simon Weidenholzer

07-68 Damian S. Damianov Jörg Oechssler Johannes Gerd Becker

07-67 Dirk Simons

Barbara E. Weißenberger
Erwartungsbildung über den Wahlausgang und ihr Einfluss auf die Wahlentscheidung

Interdependencies between Elements of Governance and Auditing: Evidence from Germany

How Does Fair Value Measurement under IAS 39 Affect Disclosure Choices of European Banks?

Managerial Optimism and Corporate Investment: Is the CEO Alone Responsible for the Relation?

IAS 39 and Biases in the Risk Perception of Financial Instruments

Continuous and Step-level Pay-off Functions in Public Good Games: A Conceptual Analysis

The role of power for distributive fairness

Why inexperienced investors do not learn: They do not know their past portfolio performance

Imitation and the Evolution of Walrasian Behavior: Theoretically Fragile but Behaviorally Robust

Uniform vs. Discriminatory Auctions with Variable Supply - Experimental Evidence

Die Konvergenz von externem und internem Rechnungswesen ñKritische Faktoren für die Entwicklung einer partiell integrierten Rechnungslegung aus theoretischer Sichtñ 


\begin{tabular}{|c|c|c|}
\hline Nr. & Author & Title \\
\hline $07-66$ & $\begin{array}{l}\text { Alexander Ludwig } \\
\text { Alexander Zimper }\end{array}$ & Attitude polarization \\
\hline $07-65$ & $\begin{array}{l}\text { Alexander Ludwig } \\
\text { Alexander Zimper }\end{array}$ & $\begin{array}{l}\text { A Parsimonious Model of Subjective Life } \\
\text { Expectancy }\end{array}$ \\
\hline $07-64$ & $\begin{array}{l}\text { Michael F. Meffert } \\
\text { Thomas Gschwend }\end{array}$ & $\begin{array}{l}\text { Voting for Coalitions? The Role of Coalition } \\
\text { Preferences and Expectations in Voting Behavior }\end{array}$ \\
\hline $07-63$ & $\begin{array}{l}\text { Michael F. Meffert } \\
\text { Thomas Gschwend }\end{array}$ & $\begin{array}{l}\text { Polls, Coalition Signals, and Strategic Voting: An } \\
\text { Experimental Investigation of Perceptions and } \\
\text { Effects }\end{array}$ \\
\hline $07-62$ & $\begin{array}{l}\text { Peter Dürsch } \\
\text { Maros Servátka }\end{array}$ & Risky Punishment and Reward in the Prisoner \\
\hline $07-61$ & $\begin{array}{l}\text { Wendelin Schnedler } \\
\text { Radovan Vadovic }\end{array}$ & Legitimacy of Control \\
\hline $07-60$ & Volker Stocké & $\begin{array}{l}\text { Strength, Sources, and Temporal Development of } \\
\text { Primary Effects of Families' Social Status on } \\
\text { Secondary School Choice }\end{array}$ \\
\hline $07-59$ & $\begin{array}{l}\text { Christopher Koch } \\
\text { Martin Weber } \\
\text { Jens Wüstemann }\end{array}$ & $\begin{array}{l}\text { Can Auditors Be Independent? - Experimental } \\
\text { Evidence }\end{array}$ \\
\hline $07-58$ & Sylvain Béal & PERCEPTRON VERSUS AUTOMATON\&8727; \\
\hline $07-57$ & $\begin{array}{l}\text { Sylvain Béal } \\
\text { Jacques Durieu } \\
\text { Philippe Solal }\end{array}$ & Farsighted Coalitional Stability in TU-games \\
\hline $07-56$ & $\begin{array}{l}\text { Alen Nosic } \\
\text { Martin Weber }\end{array}$ & $\begin{array}{l}\text { Determinants of Risk Taking Behavior: The role of } \\
\text { Risk Attitudes, Risk Perceptions and Beliefs }\end{array}$ \\
\hline $07-55$ & $\begin{array}{l}\text { Michael F. Meffert } \\
\text { Thomas Gschwend }\end{array}$ & $\begin{array}{l}\text { Strategic Voting under Proportional Representation } \\
\text { and Coalition Governments: A Simulation and } \\
\text { Laboratory Experiment }\end{array}$ \\
\hline $07-54$ & Klaus Fiedler & $\begin{array}{l}\text { Pseudocontingencies - A key paradigm for } \\
\text { understanding adaptive cognition }\end{array}$ \\
\hline
\end{tabular}




\begin{tabular}{|c|c|c|}
\hline Nr. & Author & Title \\
\hline $07-53$ & $\begin{array}{l}\text { Florian Kutzner } \\
\text { Peter Freytag } \\
\text { Tobias Vogel } \\
\text { Klaus Fiedler }\end{array}$ & $\begin{array}{l}\text { Base-rate neglect based on base-rates in } \\
\text { experience-based contingency learning }\end{array}$ \\
\hline $07-52$ & $\begin{array}{l}\text { Klaus Fiedler } \\
\text { Yaakov Kareev }\end{array}$ & $\begin{array}{l}\text { Implications and Ramifications of a Sample-Size } \\
\text { Approach to Intuition }\end{array}$ \\
\hline $07-51$ & Klaus Fiedler & $\begin{array}{l}\text { The Ultimate Sampling Dilemma in } \\
\text { Experience-Based Decision Making }\end{array}$ \\
\hline $07-50$ & $\begin{array}{l}\text { Jürgen Eichberger } \\
\text { David Kelsey }\end{array}$ & Ambiguity \\
\hline $07-49$ & Tri Vi Dang & Information Acquisition in Double Auctions \\
\hline $07-48$ & Clemens Kroneberg & $\begin{array}{l}\text { Wertrationalität und das Modell der } \\
\text { Frame-Selektion }\end{array}$ \\
\hline $07-47$ & $\begin{array}{l}\text { Dirk Simons } \\
\text { Nicole Zein }\end{array}$ & Audit market segmentation and audit quality \\
\hline $07-46$ & $\begin{array}{l}\text { Sina Borgsen } \\
\text { Martin Weber }\end{array}$ & $\begin{array}{l}\text { False Consensus and the Role of Ambiguity in } \\
\text { Predictions of Othersí Risky Preferences }\end{array}$ \\
\hline $07-45$ & $\begin{array}{l}\text { Martin Weber } \\
\text { Frank Welfens }\end{array}$ & $\begin{array}{l}\text { An Individual Level Analysis of the Disposition } \\
\text { Effect: Empirical and Experimental Evidence }\end{array}$ \\
\hline $07-44$ & $\begin{array}{l}\text { Martin Weber } \\
\text { Frank Welfens }\end{array}$ & $\begin{array}{l}\text { The Repurchase Behavior of Individual Investors: } \\
\text { An Experimental Investigation }\end{array}$ \\
\hline $07-43$ & $\begin{array}{l}\text { Manel Baucells } \\
\text { Martin Weber } \\
\text { Frank Welfens }\end{array}$ & $\begin{array}{l}\text { Reference Point Formation Over Time: A } \\
\text { Weighting Function Approach }\end{array}$ \\
\hline $07-42$ & $\begin{array}{l}\text { Martin Weber } \\
\text { Frank Welfens }\end{array}$ & $\begin{array}{l}\text { How do Markets React to Fundamental Shocks? An } \\
\text { Experimental Analysis on Underreaction and } \\
\text { Momentum }\end{array}$ \\
\hline $07-41$ & $\begin{array}{l}\text { Ernst Maug } \\
\text { Ingolf Dittmann }\end{array}$ & $\begin{array}{l}\text { Lower Salaries and No Options: The Optimal } \\
\text { Structure of Executive Pay }\end{array}$ \\
\hline
\end{tabular}

